\title{
Menschliche Komplexität und Psychosomatik
}

Antonio Malgaroli

Herbert George Wells, einer der ersten ScienceFiction-Autoren neben Jules Verne, behandelt in seinem Roman The History of Mr. Polly (1910) ein sehr reales Problem. Der Protagonist führt ein schwieriges Familien- und Berufsleben, in einem komplexen sozialen Umfeld in der Art von Charles Dickens, geplagt von den ständigen Auseinandersetzungen mit seiner Frau, die ihm seine mangelnde Arbeitsneigung und die zahlreichen Misserfolge nicht verzeihen konnte. Er entwickelte eine Reihe sehr seltsamer Pathologien, darunter lästige Verdauungsprobleme. Die Symptomatik würde heute als "psychosomatisch» bezeichnet, ein Begriff, der in der Medizin seit vielen Jahren zu Unrecht negativ besetzt ist. Wenn der Arzt mit mehreren Funktionsstörungen konfrontiert wird, ohne objektive anatomische Veränderungen, ohne Variationen der Labordiagnostik, kurz gesagt, wenn die Diagnose zu schwierig ist, wird der Patient als an einer psychosomatischen Störung leidend bezeichnet, sozusagen, der Patient hat nichts Wichtiges und verdient keine weitere Aufmerksamkeit. Obwohl man annimmt, dass diese Etikette auf das Werk von Sigmund Freud zurückgeht, wurde sie jedoch viele Jahre zuvor, Anfang des 19. Jahrhunderts, vom Leipziger Arzt Johann Christian Heinroth (1818) geprägt, vielleicht dem ersten Psychiater-Psychotherapeuten der Neuzeit (Steinberg, 2004). Heinroth prägte neben dem Begriff "psychosomatisch» auch den spiegelnden Begriff «somatopsychisch», mit Bezug auf jene organischen Pathologien, die Metastasen psychischer Natur zu induzieren schienen.

An dieser Stelle müssen wir kurz in ein Zeitalter zurückschalten, in dem die Medizin die Krankheit als Zustand des Unbehagens des Menschen in seiner «Ganzheit» begriff. Die alten Griechen sahen den Menschen als eine untrennbare Einheit von Psyche, vom griechischen Psyché ( $\psi v \chi \eta ́)$ und Sòma $(\sigma \tilde{\omega} \mu \alpha)$, wo Psyché für Ein- und Ausatmen, Blasen ( $\psi v ́ \chi \omega)$ also Atmen steht, implizit ein Hauch von Leben. Psyché symbolisierte für die Griechen die Seele: Nach Platon öffnet die mythologische Figur von Psyché, die Künstler aller Zeiten fasziniert hat, im Augenblick des Todes die Schmetterlingsflügel und trennt sich vom Körper und überwindet die irdische Körperlichkeit, um einen höheren, göttlichen Zustand zu erreichen.

Da sie nicht sichtbar und quantifizierbar war und nicht objektiviert werden konnte, war ihre
Subjektivität offensichtlich von aussen schwer zu bewerten; so sehr, dass Carl Jung enttäuscht erklärte, dass «die Psychologie als Wissenschaft abgeschafft werden muss und erst dann ihr Ziel erreichen kann» (Jung, 1994). Diese pessimistische Vision war von der Beharrlichkeit der Einflüsse der Theorien von Rene Descartes aus dem 18. Jahrhundert beeinflusst, die gezeigt hatten, wie das Funktionieren des Organismus und damit des Sòma (Res Extensa) den Gesetzen der Physik folgte und deshalb mathematisch messbar war. Während diese materialistische Vision die Grundlage für die Anwendung der exakten Wissenschaften auf Biologie und Medizin gelegt hatte, hatte die Unterscheidung von Psyché (Res Cogitans), die nicht in gleicher Weise objektiviert werden kann, den Beginn der Trennung zwischen Psyché und Sòma dekretiert.

Die Entwicklung der Bedeutung von Psyché und Sòma war und ist sehr komplex. Im dritten Jahrtausend können wir jedoch nicht weiter über Sòma oder den Körper sprechen, ohne Neuronen, Synapsen, Nervenfasern, das Gehirn, kurz das Nervensystem als Ganzes einzubeziehen. Das Nervensystem ist zweifellos ein Teil unseres Körpers, es reguliert ihn und wird durch ihn reguliert. Andererseits, wenn wir über Psyché als Synonym von Geist sprechen, können wir nicht ignorieren, dass das Gehirn eine ganze Reihe von Funktionen impliziert, die wir als Ganzes Geist nennen. Heute wird allgemein anerkannt, dass unser Gehirn der Sitz des organischen Teils der Psyché ist. Die derzeit angesagteste Hypothese - aber immer noch eine Hypothese - argumentiert, dass sich die Arbeit des Geistes für jede spezifische Aufgabe auf kleine Bereiche oder Hirnströme beschränkt. Geistige Funktionen wären demnach als modular einzustufen. Diese Hypothese der zerebralen Fragmentierung, von der der Phrenologe Gall ausgeht, nahm im 20. Jahrhundert dank der Charakterisierung bestimmter Arten von Hirnschäden Gestalt an; eine Hypothese, die dann durch die Studien zur zerebralen Elektrostimulation, der ersten, die der kanadische Neurochirurg Wilder Penfield durchführte, gefestigt wurde. Wenn die Hippocampus-Region beschädigt wird, verlieren wir die Fähigkeit, neue Erinnerungen zu speichern. Wenn wir eine Läsion im Broca-Areal haben, hören wir auf zu reden. Wenn die Läsion den Frontallappen betrifft, beginnen wir, riskante und oft moralisch fragwürdige Entscheidun-

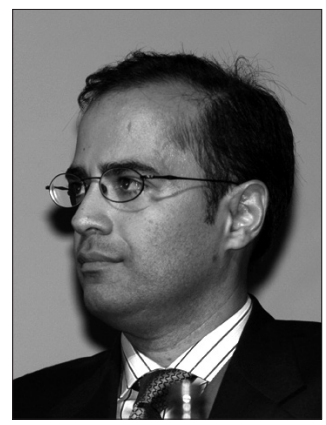


gen zu treffen. Der Neurowissenschaftler Roger Sperry zeigte 1958, dass der Abschnitt der Region, der die beiden Hemisphären verbindet, wo die Fasern, die die neokortikalen Bereiche der rechten mit denen der linken (Corpus callosum) verbinden, sogar das Erscheinen zweier organisierter und unabhängiger «Köpfe» bewirkten: Informationen, Gedanken, Gefühle, die in der einen Hemisphäre vorhanden waren, unterschieden sich und wurden für die andere Hemisphäre unzugänglich.

Sperry schrieb in diesem Zusammenhang:

"Jede linke und rechte Hemisphäre besitzt ihre eigene private Kette von Erinnerungen und Lernerfahrungen, die für die andere Hemisphäre unzugänglich sind. In vielerlei Hinsicht scheint jede getrennte Hemisphäre einen eigenen Geist zu haben.»

Sperry (1982)

Heute gilt als erwiesen, dass nach Pathologien, traumatischen oder chirurgischen Verletzungen, durch elektrische Reize, wie sie mit der Tiefen Hirnstimulation (Deep Brain Stimulation DBS; Graat et al., 2017) oder durch den Einsatz der Transkranen Magnetstimulation (TMS; Vidal-Dourado et al., 2014), aber auch mit pharmakologischen Mitteln, die auf spezifische Neurotransmitterwege einwirken, die «Psychè» eines Menschen manipuliert oder verändert werden kann. Aber künstliche Veränderungen des «Verstandes» zu induzieren, bedeutet noch nicht, seine Bedeutung, seine Mechanismen oder die Veränderung spezifischer interpretativer Codes verstanden zu haben. Wir wissen immer noch zu wenig über das Gehirn, um zu verstehen, wie der Geist derart abstrakte Funktionen wie das Erreichen des Bewusstseins seiner selbst als Individuum (Ich-Bewusstsein, Selbstbewusstsein) ausführen kann.

Wie viele unserer Schwierigkeiten und Illusionen ergeben sich aus der unglaublichen Komplexität des Geistes? Das Gehirn besteht aus vielen unabhängigen, aber sehr gut integrierten Einheiten, die auf der Grundlage spezifischer Verbindungshierarchien organisiert sind und kommunizieren. Aber wie ist es möglich, dass der Geist aus der Kombination einer einfachen neuronalen Zelle stammt, einer im Grunde unbelebten Materie, die durch den Einsatz rein physikalischer Prozesse verändert wurde? Es ist offensichtlich, dass wir es mit einem komplexen System zu tun haben, wenn wir von einer

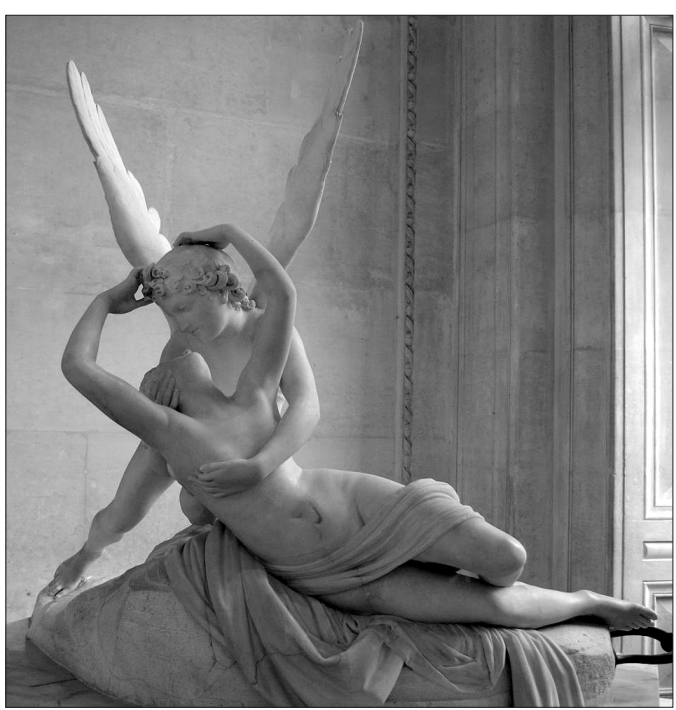

Amor und Psyché, Antonio Canova', Louvre Paris

Struktur sprechen, die etwa 1.000 Milliarden miteinander verbundene Neuronen enthält, von denen jedes mit mindestens 100.000 anderen Neuronen verbunden ist: zweifellos das komplexeste System, das je beschrieben wurde. Die organisierte Integration dieser einfachen Elemente erzeugt ein System, das sich in seinen einzelnen Teilen sehr unterschiedlich verhält. Anders ausgedrückt, dank der synaptischen Verbindungen entstehen neue Eigenschaften, neue Fähigkeiten, die es bisher nicht gab. Wir wissen, dass ein einzelnes Neuron doch nicht so einfach ist, da es selbst sehr komplexe Aufgaben ausführen kann (Koch \& Laurent, 1999), aber um einen denkenden Geist zu bekommen, ist es wahrscheinlich, dass man eine kritische Anzahl von Zellen überwinden muss. In einem derartigen System sind die Schaltkreise und die synaptischen Beziehungen zwischen den Modulen viel wichtiger als die Module selbst. Ähnlich wie in den Tiergesellschaften, in denen der Einzelne unterworfen und oft den Bedürfnissen der Gemeinschaft geopfert wird. Nur auf diese Weise kann die Gruppe Dinge tun, die der Einzelne nie erreichen könnte. Tatsächlich hängt die Komplexität des Geistes nicht so sehr von der Anzahl der vorhandenen neuronalen Zellen, sondern von der Anzahl der Verbindungen zwischen ih-

1 Zu beachten ist, dass Antonio Canova (1757-1822) im Gegensatz zu den klassischen hellenistischen und römischen Darstellungen Psyché ohne die Flügel eines Schmetterlings darstellt. 
nen ab, was ein Hinweis auf die Kommunikationsfähigkeit des Systems ist. Ein System dieser Komplexität sollte auf den ersten Blick nicht von der Eliminierung einer Reihe seiner Elemente betroffen sein. Das System funktioniert weiter, wenn ein Modul entfernt oder beschädigt wird, aber seine Eigenschaften und Merkmale ändern sich, wie wir oben erläutert haben. Wenn wir die rechte Gehirnhälfte (Parietallappen) entfernen, wird das Gehirn nicht mehr in der Lage sein, die kontralaterale Hälfte seines Körpers und des umgebenden Raumes zu erkennen (Neglet-Syndrom; Bisiach \& Luzzatti, 1978). Gedanken, Ideen, Ängste, Überzeugungen, Motivationen, Erinnerungen werden jedoch bleiben.

In diesem Zusammenhang ist es sehr interessant zu beobachten, dass in einem einfachen System eine synaptische Übertragung, die aus einigen wenigen Elementen und Verbindungen besteht, stabil ist und geordnete und sehr vorhersehbare Ergebnisse liefert. Steigt dagegen die Anzahl der Zellen und Verbindungen zu stark an, löst sich das System auf, wird laut, chaotisch und beginnt lawinenartig Ereignisse zu zeigen, die eine Vielzahl von Modulen gleichzeitig einbeziehen. Diese lawinenartigen Ereignisse führen dazu, dass die konstituierenden Elemente ihre Unabhängigkeit verlieren, und dieser Mangel an Unabhängigkeit reduziert die gesamte Rechenleistung. Der interessanteste und produktivste Zustand liegt offensichtlich zwischen Ordnung und Unordnung. Genau auf dieser Stufe offenbart das neuronale System eine maximale Anzahl von unterschiedlichen Verhaltensweisen und Fähigkeiten. Man muss nicht davon ausgehen, dass diese Verhaltensweise nur bei makroskopischen Systemen beobachtet wird. In Wahrheit hängt sie nur von der intrinsischen Komplexität des Systems ab und kann auch in mikroskopischen Strukturen, wie beispielsweise Synapsen, erzeugt werden. Synapsen, Strukturen von einigen hundert Milliardstel Metern, die zwischen geordneten und chaotischen Zuständen oszillieren (Lamanna \& Malgaroli, 2015), können aus Erfahrungen lernen (Malgaroli et al., 1995). Der interessanteste und produktivste Zustand liegt offensichtlich zwischen Ordnung und Unordnung. Eine grosse Anzahl von möglichen unterschiedlichen Verhaltensweisen, d.h. die Fähigkeit zur Diversifizierung, ist eine grundlegende Eigenschaft, um sich an die Umgebung anzupassen, sich zu verändern und so zu überleben.
Kann ein Psychologe demnach vorhersagen, wie sich ein Patient fühlt, ohne auf ihn zu hören, ohne darüber nachzudenken, was er sagt, ohne mit ihm zu sprechen, ohne sich um ihn als Ganzes zu kümmern? Können wir vorhersagen, wie sich ein Subjekt beim Hören eines Musikstücks fühlen wird? Es gibt Menschen, die sich glücklich fühlen, andere reagieren bewegt und weinen. Einige hören interessiert zu, andere achten nicht darauf oder sind einfach nur irritiert. Woher kommt diese enorme Vielfalt, die das Verständnis und die Interpretation des menschlichen Fühlens und Empfindens so schwer macht? Die grosse Variabilität möglicher Verhaltensreaktionen hängt von genetischen und epigenetischen Faktoren ab. Aber in erster Linie beruht sie auf unseren bisherigen Erfahrungen, unserem aktuellen emotionalen Zustand, auf kontingenten Motivationen. Die jüngsten neurowissenschaftlichen Untersuchungen bestätigen, was wir oben ausgeführt haben. Die Erfahrungen verändern Anzahl und räumliche Verteilung der synaptischen Verbindungen im Gehirn (Holtmaat \& Svoboda, 2009); ein Prozess, der die Unvorhersehbarkeit der Zustände unseres Geistes erhöht und ihn vom Geist eines anderen Individuums differenziert. Die Ereignisse unseres Innenlebens, seit jeher Gegenstand der Analyse durch Psychologie, Psychoanalyse, Psychiatrie, sind offensichtlich auftauchende Eigenschaften elementarer Gehirnprozesse, deren Schichtung im Laufe des Lebens von unseren persönlichen Erfahrungen geleitet wird. Was heute in einer so materialistisch gewordenen Welt der Forschung fehlt, ist das Verständnis, dass diese vielfältigen Prozesse auf eine andere Weise untersucht und verstanden werden müssen. Eigentlich war Jung zu Recht entmutigt (Jung, 1994). Man kann nicht den gleichen Massstab der Beurteilung und die gleichen Vorgehensweisen verwenden, um elementare Prozesse zu untersuchen, zum Beispiel die klassischen Prozesse der Anatomie, Physiologie und Pharmakologie, auch wenn diese im Laufe der Jahre zu unglaublichen Ergebnissen geführt haben. Die komplexen Prozesse des Geistes folgen ihren eigenen Gesetzen und haben sehr unterschiedliche Dynamiken und Eigenschaften in Bezug auf die konstituierenden Elemente. Es wäre daher einfach nur illusorisch zu glauben, dass wir den gleichen Massstab der Analyse oder Beurteilung der deterministischen Wissenschaften anwenden könnten. Innere Vor- 
gänge, die sich von Mensch zu Mensch unterscheiden, können sicherlich nicht auf banale Variationen der elementaren elektrischen Aktivität reduziert werden, die in einigen Subpopulationen von neuronalen Zellen zu bestimmten Zeiten stattfinden. Nicht einmal in tausend Jahren wird es möglich sein zu verstehen, was ein Individuum denkt oder fühlt, ohne mit ihm zu sprechen, ohne unbewusste Erfahrungen, Gedanken oder Ängste an die Oberfläche zu bringen, die zwar physisch im Gehirn kodiert sind, deren Auswirkungen jedoch, aufgrund der inneren Komplexität, schwierig zu bewerten sind. Verantwortlich dafür sind die komplexen Phänomene der Resonanz mit der Psyché (Seele) derjenigen, die sie beherbergen.

Ebenfalls im Kontext einer integrierten Vision von Psyché und Sòma ist Homöostase ein sehr wichtiger Aspekt. Unabhängige Einheiten müssen auf verschiedenen Ebenen, nach genauen Hierarchien innerhalb des Gehirns, aber auch zwischen Gehirn und Körper und umgekehrt kommunizieren, um in direktem Kontakt mit der Umwelt innerhalb und ausserhalb des Sòma zu bleiben. Diese ständige Aktivität erfordert mehrere Mechanismen der Signaltransduktion und eine rasche Behebung von Änderungen und Fehlfunktionen. Ziel ist es, das System wieder auf den Stand der maximalen kommunikativen Funktionalität zu bringen. Der französische Physiologe Claude Bernard (Conti, 2013) postulierte um die Mitte des 19. Jahrhunderts die Existenz eines «milieu intérieur» (Bernard, 1859), einer von allen Zellen geteilten, zur Selbstregulierung fähigen inneren Umgebung. Dieses Milieu Intérieur gibt es tatsächlich; heute nennen wir es «extrazelluläre Flüssigkeit». Es handelt sich um eine komplexe Flüssigkeit, im Gleichgewicht mit anderen Körperflüssigkeiten, die alle Zellen unseres Körpers verbindet. Dies geschieht durch Signalmoleküle wie Hormone, Neurotransmitter, Metaboliten, Ionen, Proteine, Antikörper, Immunzellen oder kleine Nukleotidsequenzen. Es ist wichtig zu unterstreichen, wie das Leben der Zellen, ihre Aktivität, ihr Stoffwechsel und ihre synaptischen Kommunikationsprozesse dieses Milieu Intérieur grundlegend verändern und seine ionische Zusammensetzung, Temperatur, den Säuregehalt und den Gehalt an
Neurotransmittern und Metaboliten verändern (Bernard, 1859).

Homöostatische Prozesse müssen daher die Ausgangsbedingungen dieser gemeinsamen Flüssigmatrix sehr schnell wiederherstellen. Wenn wir zu diesen Überlegungen die Tatsache hinzufügen, dass das sehr dichte Netz neuronaler Fasern, das praktisch alle Organe und Gewebe unseres Organismus erreicht, aber auch die Neuronen, die an peripheren Vorposten wie dem Darm stationiert sind, in dieses Milieu Intérieur getaucht sind, verstehen wir die grundlegende Rolle dieser homöostatischen Regulierprozesse. Umgekehrt können wir die enormen Auswirkungen auf Medizin und Psychologie all jener Bedingungen nachvollziehen, unter denen sich diese Mechanismen als gestört erweisen.

Diese kurzen Ausführungen und die tiefen Verbindungen, von denen wir heute wissen, dass sie zwischen Psyché und Sòma bestehen, helfen uns, zu verstehen, wie sehr die sogenannten psychosomatischen Störungen konkret und schädlich für den betroffenen Menschen sind. Die Probleme des täglichen Lebens, emotionaler Stress, Konflikte wie diejenigen, mit denen Herr Polly konfrontiert war, erzeugen dysregulatorische Phänomene, die zunächst Funktionsstörungen und später richtige organische Krankheiten verursachen können. In einigen Fällen können sie zu vollkommen unerklärlichen Todesfällen führen. Oft sind Personen $\mathrm{zu}$ beobachten, die durch starken emotionalen Stress innerhalb weniger Tage graue Haare bekommen oder sich mit zahlreichen Xanthelasmen konfrontiert sehen. Es sind Anzeichen auf eine schwere Stoffwechselstörung, die Phänomene der Immunsuppression mit Auslösung schwerer Krankheiten entwickeln können. Im dritten Jahrtausend macht es keinen Sinn mehr, psychosomatische Erkrankungen als Nicht-Erkrankungen zu behandeln. Die Abgrenzung zwischen Medizin, Psychoanalyse, Psychosomatik ist heute sehr künstlich, so wie es die Geist-Körper-Dichotomie des kartesischen Dualismus war. Die Zukunft gehört einer anthropologischen, ganzheitlichen Vision des Menschen und seiner Krankheiten. Sie muss in der Lage sein, dem Menschen in seinen täglichen Schwierigkeiten durch ein wahres Verständnis der Schnittstellen zwischen Psyché und Sòma zu helfen. 


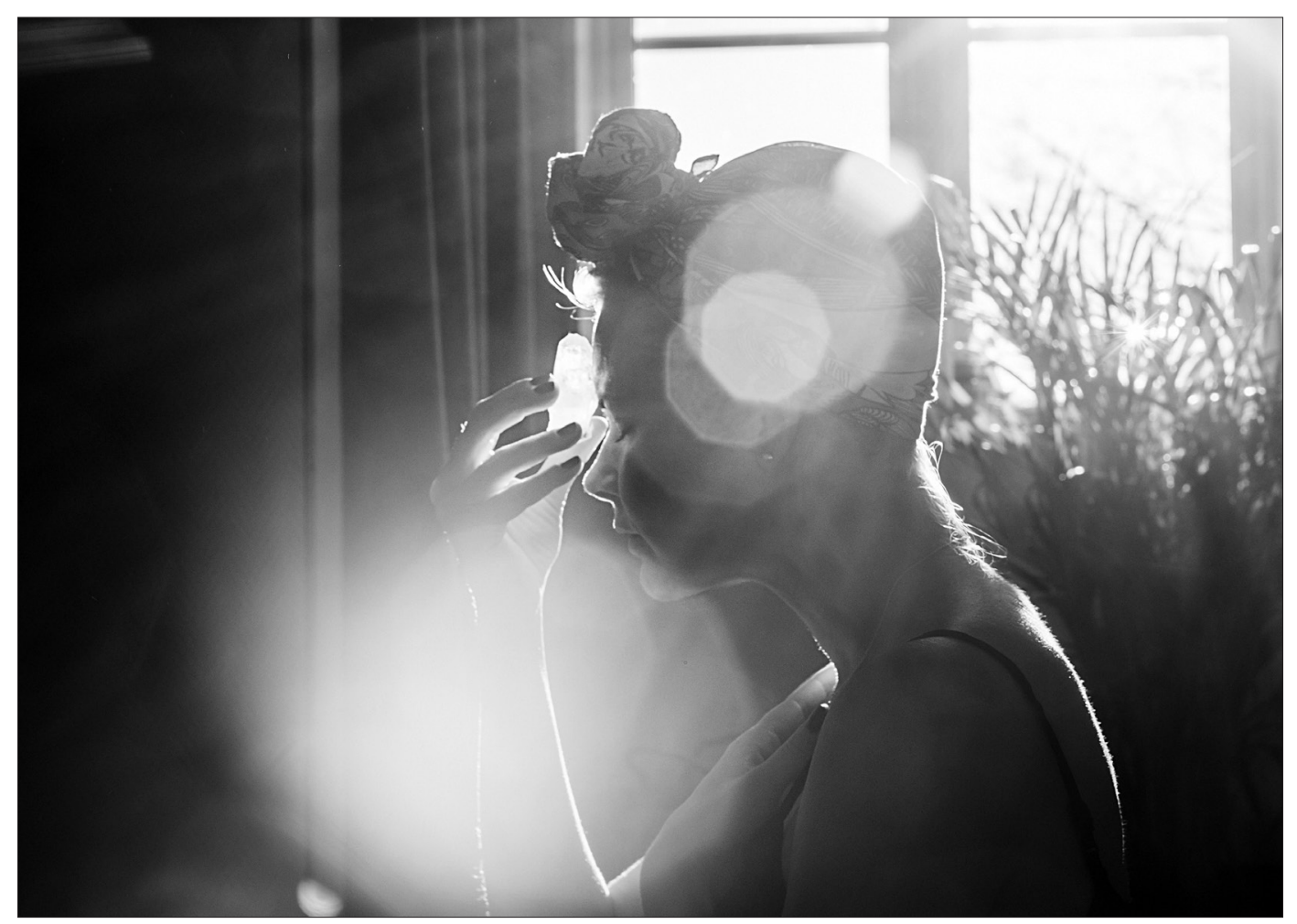

\section{Literatur}

Bernard, C. (1859). Lecons sur le proprietes physiologiques et les alterations pathologiques des liquides de l’organisme. Paris: Bailliere.

Bisiach, E. \& Luzzatti, C. (1978). Unilateral neglect of representational space. Cortex, 14(1), 129-33.

Conti, F. (2013). Claude Bernard e la nascita della biomedicina. Milano: Cortina editore.

Graat, I., Figee, M. \& Denys, D. (2017). The application of deep brain stimulation in the treatment of psychiatric disorders. International Review Psychiatry, 29(2), 178-190.

Heinroth, J.C. A. (1818). Lehrbuch der Störungen des Seelenlebens oder der Seelenstörungen und ihrer Behandlung. Leipzig: Vogel.

Holtmaat, A. \& Svoboda, K. (2009). Experience-dependent structural synaptic plasticity in the mammalian brain. Nature Review Neuroscience, 10(9), 647-58.

Jung, C.G. (1994). Riflessioni Teoriche sull'essenza della psiche. In C. G. Jung, La dinamica dell'inconscio. Torino: Bollati Boringhieri.

Koch. C. \& Laurent, G. (1999). Complexity and the nervous system. Science, 284(5411), 96-8.
Lamanna, J. \& Malgaroli, A. (2015). A pre-docking source for the power-law behavior of spontaneous quantal release: application to the analysis of LTP. Frontiers Cellular Neuroscience, 18(9): 44-54.

Malgaroli, A., Ting, A. E., Wendland, B., Bergamaschi, A., Villa, A., Tsien, R. W. \& Scheller R.H. (1995). Presynaptic component of long-term potentiation visualized at individual hippocampal synapses. Science, 268(5217), 1624-8.

Sperry, R. (1982). Some effects of disconnecting the cerebral heispheres. Nobel Lecture, 8. December 1981. Bioscience Reports, 2(5), 265-276.

Steinberg, H. (2004). Die Errichtung des ersten psychiatrischen Lehrstuhls: Johann Christian August Heinroth in Leipzig, Nervenarzt. 75(3), 303-7.

Vidal-Dourado, M., Conforto, A.B., Caboclo, L. O., Scaff, M., Guilhoto, L. M. \& Yacubian, E. M. (2014). Magnetic fields in noninvasive brain stimulation. Neuroscientist, 20(2), 112-21.

Wells, H.G. (1910). The History of Mr. Polly. New York: Thomas Nelson and Son.

Antonio Malgaroli ist Psychiater und Professor für Physiologie und Neurowissenschaften. 